\title{
Editorial
}

\section{The diagnosis and management of pre-invasive breast disease - current challenges, future hopes}

\section{Sunil R Lakhani}

\author{
The Breakthrough Toby Robins Breast Cancer Research Centre, ICR and The Royal Marsden Hospital, London, UK \\ Correspondence: Sunil R Lakhani (e-mail: Sunil.Lakhani@icr.ac.uk) \\ Published: 28 July 2003 \\ Breast Cancer Res 2003, 5:248-249 (DOI 10.1186/bcr641) \\ (C) 2003 BioMed Central Ltd (Print ISSN 1465-5411; Online ISSN 1465-542X) \\ Keywords: breast, classification, DCIS, molecular, LCIS
}

\section{Introduction}

The dogma of cancer biology is that tumours arise in a multistep fashion, and colon cancer has been the paradigm of this type of model. It is no surprise that the same model has been adopted in other cancer types, and breast cancer is no exception. Invasive breast cancer is believed to arise via a series of intermediate lesions, which are currently classified as hyperplasias with and without atypia, and in situ carcinomas.

The multistep model has also formed the basis for the screening programmes instituted for cervical and breast cancer. If tumours arise via intermediate steps and it is possible to identify one or more of these steps, it becomes possible to treat the patient before the tumour has had a chance to metastasise.

Whether the screening programme has reduced the mortality from breast cancer is a controversial issue. The consensus view seems to be that it has had an impact on mortality, although the level of reduction is hotly debated. Whatever prejudices one holds, the fact remains that with the introduction of the screening programme there has been an increase in the detection of pre-invasive breast disease, and this has highlighted deficiencies in the classification systems and the lack of knowledge regarding the natural history of the lesions. This lack of knowledge has led to significant problems in diagnosis and management of patients with proliferative breast disease, so that patients are offered diverse treatment options ranging from 'nothing' to mastectomy.

The articles in the present thematic review series were conceived to highlight the problems faced by doctors and scientists dealing with pre-invasive breast disease, and to throw light on the evolving classification systems and molecular techniques that are likely to change the way we approach patients with these disorders.
The series will be published in two parts, the first half in the present issue of Breast Cancer Research and the rest of the papers in the following issue.

Andy Evans kicks off by describing the current challenges in the radiological diagnosis of pre-invasive lesions [1]. This is followed by a series of papers dealing with the pathological classification. Sarah Pinder and lan Ellis discuss ductal carcinoma in situ and atypical ductal hyperplasia [2], and Peter Simpson and colleagues describe the current status with lobular carcinoma in situ [3]. Recently, as a direct result of identifying calcification on mammography, pathologists have been encountering a range of proliferations, which have been described by an array of names including columnar cell lesions/flat epithelial atypia. Stuart Schnitt has summarised the current classification and management issues relating to diagnosis of these lesions on core biopsies [4]. Should the patient with flat atypia on a core biopsy have a further excision? Marc Van der Vijver and Hans Peterse finish off the first half of the series with their own views and concerns regarding the existing terminology for classification, and provide a personal approach (which may not be shared by all) as to how they deal with the problem in their own practice [5].

In the Breast Cancer Research issue to follow, Ashutosh Nerurkar and Peter Osin discuss the new techniques of core biopsy and ductal lavage in diagnosis [6], and Arnie Purushotham describes the surgical approach to management of patients with early disease [7]. The last two articles in the series deal with molecular techniques used to study pre-invasive breast lesions. Jorge Reis Filho and Sunil Lakhani review the current data on genomic alteration in hyperplasias and in situ carcinomas [8], and Stefanie Jeffrey and Jonathan Pollack discuss how the new and emerging technologies such as microarrays will impact the understanding of these pre-cancerous proliferations [9]. 
The series of articles in these reviews intends to educate as well as to raise questions about our knowledge, prompting doctors and scientists to come together to meet the challenge. It is hoped that this will make a difference to the management of patients with pre-invasive breast disease.

\section{Competing interests}

None declared.

\section{References}

1. Evans A: The diagnosis and management of pre-invasive breast disease: Radiological diagnosis. Breast Cancer Res 2003, 5:250-253.

2. Pinder SE, Ellis IO: The diagnosis and management of preinvasive breast disease: Ductal carcinoma in situ (DCIS) and atypical ductal hyperplasia (ADH) - current definitions and classification. Breast Cancer Res 2003, 5:254-257.

3. Simpson PT, Gale T, Fulford LG, Reis-Filho JS, Lakhani SR: The diagnosis and management of pre-invasive breast disease: Pathology of atypical lobular hyperplasia and lobular carcinoma in situ. Breast Cancer Res 2003, 5:258-262.

4. Schnitt SJ: The diagnosis and management of pre-invasive breast disease: Flat epithelial atypia - classification, pathologic features and clinical significance. Breast Cancer Res 2003, 5:263-268.

5. Van de Vijver MJ, Peterse $\mathrm{H}$ : The diagnosis and management of pre-invasive breast disease: Pathological diagnosis - problems with existing classifications. Breast Cancer Res 2003, 5:269-275.

6. Nerurkar A, Osin P: The diagnosis and management of preinvasive breast disease: The role of new diagnostic techniques. Breast Cancer Res, in press.

7. Purushotham AD: The diagnosis and management of pre-invasive breast disease: Problems associated with management of pre-invasive lesions. Breast Cancer Res, in press.

8. Reis-Filho JS, Lakhani SR: The diagnosis and management of pre-invasive breast disease: Genetic alterations in pre-invasive lesions. Breast Cancer Res, in press.

9. Jeffrey SS, Pollack JR: The diagnosis and management of preinvasive breast disease: The promise of new technologies in understanding pre-invasive lesions. Breast Cancer Res, in press.

\section{Correspondence}

Sunil R Lakhani, The Breakthrough Toby Robins Breast Cancer Research Centre, Mary-Jean Mitchell Green Building, Chester Beatty Laboratories, ICR, Fulham Road, London SW3 6JB, UK. Tel: +44 20

7153 5525; fax: +44 207153 5533; e-mail: Sunil.Lakhani@icr.ac.uk 